\title{
A blood boost for NAD+
}

Dyslipidemia, or abnormally high levels of fats and cholesterol in the blood, can lead to one of several lifestyle-related illnesses due to overconsumption of unhealthy foods. These illnesses continue to be the leading cause of disease and death in developed countries, and therefore, significant resources have been dedicated to understanding and treating the underlying causes. One area of growing interest is the use of Nicotinamide riboside (NR), a form of vitamin $\mathrm{B}_{3}$ that is a precursor to nicotinamide adenine dinucleotide (NAD+). $\mathrm{NAD}+$ is a co-enzyme found in all living cells and is involved in redox reactions during metabolism, and as such, can play a potential role in a wide variety of cellular processes and disease states. However, biomedical studies have honed in on clines in NAD+ levels as an important step in tissues during aging and overnutrition. NR has been demonstrated to increase levels $\mathrm{NAD}+$ and in certain model organisms (e.g. yeast and mice) to help extend life and improve outcomes of metabolic disease. However, in order to take advantage of NR technologies to improve treatments in humans, knowledge of in vivo pharmacokinetics and bioavailability are required.

In a recent Nature Communications article (Nat. Commun. 10, 12948; 2016) Charles Brenner and colleagues at the Carver College of Medicine, University of Iowa (Iowa City, IA), studied the oral bioavailability of NR and its utilization in different tissues using data from humans and mice. As a pilot-study, the team began by analyzing NAD+ metabolomics of blood and urine from a healthy 52-year-old man given a $1,000 \mathrm{mg}$ dose of NR daily for 7 days. They found that NAD+ levels rose 2.7 fold in the blood after a single dose, and surprisingly that NA adenine dinucleotide (NAAD) increased 45-fold. Follow-up experiments in mice further showed that NR boosts NAD+ greater than NA, and

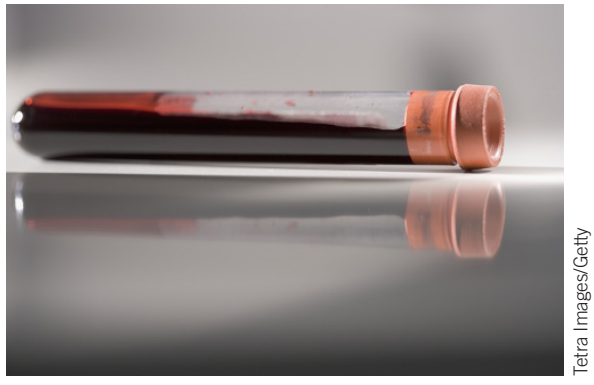

that NR is a direct precursor of NAAD. A validation clinical study in 12 healthy human subjects confirmed that NR can safely boost NAD+ levels, and also that NAAD levels serve as a sensitive biomarker of increased levels of NAD+. This unique combination of pre-clinical and clinical science demonstrates the oral bioavailability of NR, and helps establish criteria for further translation of NR treatment for human disease.

Dustin M. Graham

\section{TURNING UP THE HEAT ON THERMOREGULATION RESEARCH}

Keeping cool on a warm day is not difficult for modern humans, but over the course of evolution many organisms have struggled to survive in frequently-changing temperatures. They've developed thermoregulation, a biological process allowing for regulation of body temperature towards homeostasis. A small region of the brain's hypothalamus begins this vital regulation by detecting environmental temperature, then induces physiological changes that adjust the animal's core temperature. Unfortunately, the specific neurons that initially detect environmental temperature and kick-start this thermoregulation process have eluded researchers. However, a recent report by Zachary Knight's lab at UCSF (Cell 167, 47-59; 2016) describes novel findings of molecularly-defined warm-sensitive neurons (WSNs) in the hypothalamus of mice, presenting new methods to help understand the process of thermoregulation.

The UCSF team began their study by searching for molecular markers of WSNs. After placing mice in a warm environment ( $\left.36^{\circ} \mathrm{C}\right)$, the researchers took advantage of ribosomal changes in neurons after activation to specifically examine the mRNA sequence of these cells. They observed two genes that were highly co-expressed in cells activated by warmth, providing the first genetic access to WSNs of the hypothalamus.

Next, the team sought to understand the function of these cells in vivo. They injected a virus containing a calcium reporter into the hypothalamus of mice and used fiber photometry to observe the activity of WSNs. When the mice were placed in a temperaturechanging chamber the fiber photometry showed that temperature increases were tightly coupled with WSN activity. The authors also used their genetic access to express a specialized channel opsin in WSNs for direct control over the neuron's activity. The researchers observed that direct activation of the WSNs caused a quick decline in core body temperature of the mice, simultaneous changes in thermoregulation behavior like tail vasodilation, and even affected the animal's nest building behaviors. These are all actions associated with a bodily desire to cool down. Thus, the animals were apparently perceiving the heat associated with WSN activation, even when the environmental temperature was held at normal levels.

This finding provides a novel method of controlling the core body temperature and behaviors of animals, along with a new path of research into thermoregulation. But the methods could also be translated into clinical applications, eventually providing doctors and veterinarians new and valuable techniques for controlling a patient's body temperature.

James E. Niemeyer 